\section{TATRA \\ MOUNTaiNS \\ Mathematical Publications}

DOI: $10.2478 /$ tmmp-2020-0008

Tatra Mt. Math. Publ. 75 (2020), 121-134

\title{
NECESSARY AND SUFFICIENT CONDITIONS FOR OSCILLATORY AND ASYMPTOTIC BEHAVIOUR OF SOLUTIONS \\ TO SECOND-ORDER NONLINEAR NEUTRAL \\ DIFFERENTIAL EQUATIONS \\ WITH SEVERAL DELAYS
}

\author{
Shyam Sundar SAntra \\ Department of Mathematics, JIS College of Engineering, Kalyani-741235, Nadia, West Bengal, \\ INDIA
}

\begin{abstract}
In this paper, necessary and sufficient conditions are obtained for oscillatory and asymptotic behavior of solutions to second-order nonlinear neutral delay differential equations of the form

$\frac{\mathrm{d}}{\mathrm{d} t}\left[r(t)\left[\frac{\mathrm{d}}{\mathrm{d} t}(x(t)+p(t) x(t-\tau))\right]^{\alpha}\right]+\sum_{i=1}^{m} q_{i}(t) H\left(x\left(t-\sigma_{i}\right)\right)=0 \quad$ for $t \geq t_{0}>0$,

under the assumption $\int^{\infty}(r(\eta))^{-1 / \alpha} \mathrm{d} \eta=\infty$. Our main tool is Lebesque's dominated convergence theorem. Further, some illustrative examples showing the applicability of the new results are included.
\end{abstract}

\section{Introduction}

Consider the second-order nonlinear neutral delay differential equations of the form

$$
\frac{\mathrm{d}}{\mathrm{d} t}\left[r(t)\left[\frac{\mathrm{d}}{\mathrm{d} t}(x(t)+p(t) x(t-\tau))\right]^{\alpha}\right]+\sum_{i=1}^{m} q_{i}(t) H\left(x\left(t-\sigma_{i}\right)\right)=0,
$$

where $\alpha$ is the quotient of two odd positive integers, $\sigma_{i}$ are positive constants, $q_{i}, r, p \in C(\mathbb{R}, \mathbb{R})$ with $q_{i}(t) \geq 0$ and $r(t)>0$ for $i=1,2, \ldots, m$ and $t \geq 0$.

(C) 2020 Mathematical Institute, Slovak Academy of Sciences.

2010 Mathematics Subject Classification: $34 \mathrm{C} 10,34 \mathrm{C} 15,35 \mathrm{~K} 40$.

Keyw ords: oscillation, non-oscillation, neutral, delay, nonlinear, Lebesgue's dominated convergence theorem, Banach's contraction principle.

Licensed under the Creative Commons Attribution-NC-ND4.0 International Public License. 
We use the following assumptions:

(A1) $H \in C(\mathbb{R}, \mathbb{R}), H$ is strictly increasing and $u H(u)>0$ for $u \neq 0$.

(A2) $r(t)>0$ and $\int_{0}^{\infty}(r(\eta))^{-1 / \alpha} \mathrm{d} \eta=\infty$. Letting

$$
R(t)=\int_{0}^{t}(r(\eta))^{-1 / \alpha} \mathrm{d} \eta
$$

we have $\lim _{t \rightarrow \infty} R(t)=\infty$.

(A3) Let $p \in(-1,0]$ with $-1+(2 / 3)^{1 / \alpha} \leq-a \leq p(t) \leq 0$ for $t \in \mathbb{R}_{+}$.

(A4) Let $p \in(-1,0]$ with $-1<-a \leq p(t) \leq 0$ for $t \in \mathbb{R}_{+}$.

As examples, the functions $H(u)=u^{\gamma}$ with $\gamma$ that is the quotient of two odd positive integers and $r(t)=e^{-t}$, satisfy (A1) and (A2), respectively.

The neutral differential equations find numerous applications in natural sciences and technology. For instance, they are frequently used for the study of distributed networks containing lossless transmission lines (see, e.g., [7]). Brands [2] showed that for bounded delays, the solutions to

$$
x^{\prime \prime}(t)+q(t) x(t-\tau(t))=0
$$

are oscillatory if and only if solutions to $x^{\prime \prime}(t)+q(t) x(t)=0$ are oscillatory. B a c u likova et al. 3] have studied

$$
\frac{\mathrm{d}}{\mathrm{d} t}\left[r(t) \frac{\mathrm{d}}{\mathrm{d} t}[x(t)+p(t) x(\tau(t))]\right]+q(t) H(x(\sigma(t)))=0
$$

for $0 \leq p(t) \leq p_{0}<\infty$ and (A2), and they have obtained sufficient conditions for oscillation of solutions of (1.3) through some comparison results, where the comparison results are unpredictable. D ž u r in a [6] has studied (1.3) when $0 \leq p(t) \leq p_{0}<\infty$ and (A3), and he has established sufficient condition for oscillation of solutions of (1.3) by comparison techniques. T r i p a t h y at al. 15] have considered (1.3) and established several sufficient conditions for oscillation of solutions for (1.3) by considering (C1) $H$ is odd, (C2) $Q(t)=\min \{q(t), q(\tau(t))\} \geq 0$ with $\lim _{t \rightarrow \infty} R(t)=+\infty$ and (C3) $\inf \left\{\tau^{\prime}(t): t \geq t_{0}\right\}>0$. Unlike the assumptions (C1), (C2) and (C3), by considering $Q(t)=\min \left\{q(t), q(\tau(t)) \tau^{\prime}(t)\right\}$ and $\tau^{\prime}$ is allowed to be oscillatory, Karpuz and Santra [8] have obtained several sufficient conditions for oscillatory and asymptotic behavior of solutions of

$$
\begin{array}{r}
\frac{\mathrm{d}}{\mathrm{d} t}\left[r(t) \frac{\mathrm{d}}{\mathrm{d} t}[x(t)+p(t) x(\tau(t))]\right]+\sum_{i=1}^{m} q_{i}(t) H_{i}\left(x\left(\sigma_{i}(t)\right)\right)=0 \\
\text { for } t \geq t_{0}, \quad \text { for different ranges of } p .
\end{array}
$$




\section{NECESSARY AND SUFFICIENT CONDITIONS ...}

P in elas and S antra [10] have studied necessary and sufficient conditions of

$$
\frac{\mathrm{d}}{\mathrm{d} t}(x(t)+p(t) x(t-\tau))+\sum_{i=1}^{m} q_{i}(t) H\left(x\left(t-\sigma_{i}\right)\right)=0 .
$$

W o n g [16] has studied necessary and sufficient conditions for the oscillation of solutions to

$$
(x(t)+p x(t-\tau))^{\prime \prime}+q(t) f(t-\sigma)=0,
$$

where the constant $p$ satisfies $-1<p<0$. The motivation of the present work has come from the above studies. Hence, in this work, an attempt is made to establish necessary and sufficient conditions for oscillatory and asymptotic behavior of solutions of (1.1) without making any comparison. For more information related the oscillation of solutions to this type of equations, we refer the readers to $[1,4,5,9,11-14,17,18$. Note that most publications consider only sufficient conditions, and just a few of them consider necessary and sufficient conditions.

Let $\sigma=\max \left\{\sigma_{i}: i=1, \ldots, m\right\}$, and let $T \geq \sigma$. By a solution to (1.1) we mean a function $x \in C([T-\sigma, \infty), \mathbb{R})$ such that

$$
z(t)=x(t)+p(t) x(t-\tau)
$$

$r(t) z^{\prime}(t)$ are continuously differentiable for $t \geq T$, and (1.1) is satisfied. We consider only solutions for which $\sup \{|x(t)|: t \geq 0\}>0$. A solution is called oscillatory if it has arbitrarily large zeros; otherwise, it is called non-oscillatory.

\section{Preliminaries}

LEMma 2.1. Let conditions (A1), (A2), (A3) or (A4) be satisfied and assume that $x$ is an eventually positive solution of (1.1). Then $z$ satisfies one of the following two possible cases:

$\begin{array}{lllll}\text { (C1) } z(t)<0 & z^{\prime}(t)>0 & \text { and } & \left(r(t)\left(z^{\prime}(t)\right)^{\alpha}\right)^{\prime}<0 & \text { for all large t; } \\ \text { (C2) } z(t)>0 & z^{\prime}(t)>0 & \text { and } & \left(r(t)\left(z^{\prime}(t)\right)^{\alpha}\right)^{\prime}<0 & \text { for all large t. }\end{array}$

P r o of. Suppose that there exists a $t_{1} \geq t_{0}$ such that $x(t)>0, x(t-\tau)$, and $x\left(t-\sigma_{i}\right)>0$ for $t \geq t_{1}$ and $i=1,2, \ldots, m$. From (1.1) and (A1), it follows that

$$
\left(r(t)\left(z^{\prime}(t)\right)^{\alpha}\right)^{\prime}=-\sum_{i=1}^{m} q_{i}(t) H\left(x\left(t-\sigma_{i}\right)\right)<0 \quad \text { for } t \geq t_{1} .
$$

Consequently, $\left(r(t)\left(z^{\prime}(t)\right)^{\alpha}\right)$ is nonincreasing on $\left[t_{1}, \infty\right)$. Since $r(t)>0$, and thus either $z^{\prime}(t)<0$ or $z^{\prime}(t)>0$ for $t \geq t_{2}$, where $t_{2} \geq t_{1}$. 
If $z^{\prime}(t)>0$ for $t \geq t_{2}$, then we have $(\mathrm{C} 1)$ and $(\mathrm{C} 2)$. We prove now that $z^{\prime}(t)<0$ cannot occur.

If $z^{\prime}(t)<0$ for $t \geq t_{2}$, then there exists $\varepsilon>0$ such that $r(t)\left(z^{\prime}(t)\right)^{\alpha} \leq-\varepsilon$ for $t \geq t_{2}$, which yields upon integration over $\left[t_{2}, t\right) \subset\left[t_{2}, \infty\right)$ after dividing through by $r$ that

$$
z(t) \leq z\left(t_{2}\right)-\varepsilon^{1 / \alpha} \int_{t_{2}}^{t}(r(\eta))^{-1 / \alpha} \mathrm{d} \eta \text { for } t \geq t_{2} .
$$

By virtue of condition $(A 2), \lim _{t \rightarrow \infty} z(t)=-\infty$. We consider now the following possibilities separately.

If $x$ is unbounded, then there exists a sequence $\left\{t_{k}\right\}$ such that $\lim _{k \rightarrow \infty} t_{k}=\infty$, $t_{k}-\tau \geq t_{0}$ for all sufficiently large $k$ and $\lim _{k \rightarrow \infty} x\left(t_{k}\right)=\infty$, where

By $t_{k}-\tau \leq t_{k}$,

$$
x\left(t_{k}\right)=\max \left\{x(\eta) ; t_{0} \leq \eta \leq t_{k}\right\} .
$$

$$
x\left(t_{k}-\tau\right)=\max \left\{x(\eta) ; t_{0} \leq \eta \leq t_{k}-\tau\right\} \leq \max \left\{x(\eta) ; t_{0} \leq \eta \leq t_{k}\right\}=x\left(t_{k}\right) .
$$

Therefore, for all large $k$,

$$
z\left(t_{k}\right)=x\left(t_{k}\right)+p\left(t_{k}\right) x\left(t_{k}-\tau\right) \geq\left(1+p\left(t_{k}\right)\right) x\left(t_{k}\right)>0,
$$

which contradicts the fact that $\lim _{t \rightarrow \infty} z(t)=-\infty$.

If $x$ is bounded, then $z$ is also bounded, which contradicts $\lim _{t \rightarrow \infty} z(t)=-\infty$. Hence, $z$ satisfies one of the cases (C1) and (C2). This completes the proof.

Remark 2.1. It follows from (C2) of Lemma 2.1 that there exists $\delta>0$ such that $z(t) \geq \delta$ for all large $t$.

We assume that there exists a constant $\beta$ such that $0<\beta<\alpha$ and

$$
\frac{H(u)}{u^{\beta}} \geq \frac{H(v)}{v^{\beta}}, \quad \text { for } 0<u \leq v .
$$

A typical example of a nonlinear function satisfying (2.3) is $H(x)=|x|^{\gamma} \operatorname{sgn}(x)$ with $0<\gamma<\beta$.

Remark 2.2. The condition (2.3) implies that $H(u) / u^{\beta}$ is non-increasing.

We assume that there exists $\beta>\alpha>0$ such that

$$
\frac{H(u)}{u^{\beta}} \leq \frac{H(v)}{v^{\beta}}, \quad \text { for } 0<u \leq v .
$$

A typical example of a nonlinear function satisfying (2.4) is $H(x)=|x|^{\gamma} \operatorname{sgn}(x)$ with $\beta<\gamma$.

Remark 2.3. The condition (2.4) implies that $H(u) / u^{\beta}$ is non-decreasing. 


\section{Main Results}

Theorem 3.1. Under assumptions (A1)-(A3) and (2.3), every unbounded solution of (1.1) oscillates if and only if

$$
\int_{0}^{\infty} \sum_{i=1}^{m} q_{i}(\eta) H\left(\delta^{1 / \alpha} R\left(\eta-\sigma_{i}\right)\right) \mathrm{d} \eta=+\infty, \quad \forall \delta>0 .
$$

P r o of. To prove sufficiency by contradiction, assume that $x$ is a non-oscillatory solution of (1.1). Then, there exists $t_{1} \geq t_{0}$ such that either $x(t)>0$ or $x(t)<0$ for $t \geq t_{1}$. Assume that $x(t)>0, x(t-\tau)>0$ and $x\left(t-\sigma_{i}\right)>0$ for $t \geq t_{1}$ and $i=1,2, \ldots, m$. Then we have (2.1). From Lemma 2.1, $z$ satisfies one of the cases (C1) and (C2) for $t \geq t_{2}$, where $t_{2} \geq t_{1}$. We consider each of two cases separately.

Case 1. Let $z$ satisfies $(\mathrm{C} 1)$ for $t \geq t_{2}$. As $x$ is unbounded, there exists $T \geq$ $t_{2}$ such that $x(T)=\max \left\{x(\eta): t_{2} \leq \eta \leq T\right\}$. Then, from (1.4) we have $x(T) \leq z(T)+\left\{1-(2 / 3)^{1 / \alpha}\right\} x(T-\tau)<x(T)$, which is a contradiction.

Case 2. Let $z$ satisfies $(\mathrm{C} 2)$ for $t \geq t_{2}$. Since $r(t)\left(z^{\prime}(t)\right)^{\alpha}$ is positive, nonincreasing, and

$$
z^{\prime}(t) \leq\left(r\left(t_{3}\right) / r(t)\right)^{1 / \alpha} z^{\prime}\left(t_{3}\right) \quad \text { for } t \geq t_{3}, \quad \text { where } t_{3} \geq t_{2} .
$$

Integrating this inequality, we have

$$
z(t) \leq z\left(t_{3}\right)+\left(r\left(t_{3}\right)\right)^{1 / \alpha} z^{\prime}\left(t_{3}\right)\left(R(t)-R\left(t_{3}\right)\right) .
$$

Since $\lim _{t \rightarrow \infty} R(t)=\infty$, there exists $\delta>0$ and $t_{4} \geq t_{3}$ such that

$$
z(t) \leq \delta^{1 / \alpha} R(t) \text { for } t \geq t_{4} \text {. }
$$

Note that $\delta$ depends on the solution $x$ evaluated at a time $t_{4}$. Thus condition (3.1) must include all possible $\delta$ 's.

Upon using $z(t) \leq x(t)$, (3.2) and by assumption (2.3), we have

$$
\begin{aligned}
H\left(x\left(t-\sigma_{i}\right)\right) \geq H\left(z\left(t-\sigma_{i}\right)\right)=\frac{H\left(z\left(t-\sigma_{i}\right)\right)}{z^{\beta}\left(t-\sigma_{i}\right)} & z^{\beta}\left(t-\sigma_{i}\right) \\
& \geq \frac{H\left(\delta^{1 / \alpha} R\left(t-\sigma_{i}\right)\right)}{\left(\delta^{1 / \alpha} R\left(t-\sigma_{i}\right)\right)^{\beta}} z^{\beta}\left(t-\sigma_{i}\right) .
\end{aligned}
$$

Integrating (1.1) from $t$ to $\infty$, we have

$$
\lim _{A \rightarrow \infty}\left[r(\eta)\left(z^{\prime}(\eta)\right)^{\alpha}\right]_{t}^{A}+\int_{t}^{\infty} \sum_{i=1}^{m} q_{i}(\eta) \frac{H\left(\delta^{1 / \alpha} R\left(\eta-\sigma_{i}\right)\right)}{\left(\delta^{1 / \alpha} R\left(\eta-\sigma_{i}\right)\right)^{\beta}} z^{\beta}\left(\eta-\sigma_{i}\right) \mathrm{d} \eta \leq 0 .
$$


Using that $r(t)\left(z^{\prime}(t)\right)^{\alpha}$ is positive and non-increasing, we have

$$
\int_{t}^{\infty} \sum_{i=1}^{m} q_{i}(\eta) \frac{H\left(\delta^{1 / \alpha} R\left(\eta-\sigma_{i}\right)\right)}{\left(\delta^{1 / \alpha} R\left(\eta-\sigma_{i}\right)\right)^{\beta}} z^{\beta}\left(\eta-\sigma_{i}\right) \mathrm{d} \eta \leq r(t)\left(z^{\prime}(t)\right)^{\alpha} \quad \text { for } t \geq t_{4} .
$$

Therefore,

$$
z^{\prime}(t) \geq\left[\frac{1}{r(t)} \int_{t}^{\infty} \sum_{i=1}^{m} q_{i}(\eta) \frac{H\left(\delta^{1 / \alpha} R\left(\eta-\sigma_{i}\right)\right)}{\left(\delta^{1 / \alpha} R\left(\eta-\sigma_{i}\right)\right)^{\beta}} z^{\beta}\left(\eta-\sigma_{i}\right) \mathrm{d} \eta\right]^{1 / \alpha}
$$

Integrating from $t_{4}$ to $t$, we obtain

$$
\begin{aligned}
z(t)-z\left(t_{4}\right) ! & \geq \int_{t_{4}}^{t}\left[\frac{1}{r(\eta)} \int_{\eta}^{\infty} \sum_{i=1}^{m} q_{i}(\zeta) \frac{H\left(\delta^{1 / \alpha} R\left(\zeta-\sigma_{i}\right)\right)}{\left(\delta^{1 / \alpha} R\left(\zeta-\sigma_{i}\right)\right)^{\beta}} z^{\beta}\left(\zeta-\sigma_{i}\right) \mathrm{d} \zeta\right]^{1 / \alpha} \mathrm{d} \eta \\
& \geq \int_{t_{4}}^{t}\left[\frac{1}{r(\eta)} \int_{t}^{\infty} \sum_{i=1}^{m} q_{i}(\zeta) \frac{H\left(\delta^{1 / \alpha} R\left(\zeta-\sigma_{i}\right)\right)}{\left(\delta^{1 / \alpha} R\left(\zeta-\sigma_{i}\right)\right)^{\beta}} z^{\beta}\left(\zeta-\sigma_{i}\right) \mathrm{d} \zeta\right]^{1 / \alpha} \mathrm{d} \eta
\end{aligned}
$$

Letting

$$
w(t)=\int_{t}^{\infty} \sum_{i=1}^{m} q_{i}(\zeta) \frac{H\left(\delta^{1 / \alpha} R\left(\zeta-\sigma_{i}\right)\right)}{\left(\delta^{1 / \alpha} R\left(\zeta-\sigma_{i}\right)\right)^{\beta}} z^{\beta}\left(\zeta-\sigma_{i}\right) \mathrm{d} \zeta,
$$

from the above inequality, and since $z\left(t_{4}\right)>0$, we have

$$
z(t)>\left(R(t)-R\left(t_{4}\right)\right) w^{1 / \alpha}(t) .
$$

Because $\lim _{t \rightarrow \infty} R(t)=\infty$, there exists $t_{5} \geq t_{4}$ such that

$$
R(t)-R\left(t_{4}\right) \geq \frac{1}{2} R(t) \quad \text { for } t \geq t_{5} .
$$

Then

$$
z(t)>\frac{1}{2} R(t) w^{1 / \alpha}(t) \quad \text { for } t \geq t_{5},
$$

and $z^{\beta} /\left(\delta^{1 / \alpha} R\right)^{\beta} \geq w^{\beta / \alpha} /\left(2 \delta^{1 / \alpha}\right)^{\beta}$. Taking the derivative we have

$$
\begin{aligned}
w^{\prime}(t) & =-\sum_{i=1}^{m} q_{i}(t) \frac{H\left(\delta^{1 / \alpha} R\left(t-\sigma_{i}\right)\right)}{\left(\delta^{1 / \alpha} R\left(t-\sigma_{i}\right)\right)^{\beta}} z^{\beta}\left(t-\sigma_{i}\right) \\
& \leq-\sum_{i=1}^{m} q_{i}(t) H\left(\delta^{1 / \alpha} R\left(t-\sigma_{i}\right)\right) w^{\beta / \alpha}\left(t-\sigma_{i}\right) \frac{1}{\left(2 \delta^{1 / \alpha}\right)^{\beta}} \leq 0 .
\end{aligned}
$$

Therefore, $w(t)$ is non-increasing so $w^{\beta / \alpha}\left(t-\sigma_{i}\right) / w^{\beta / \alpha}(t) \geq 1$, and $\left(w^{1-\beta / \alpha}(t)\right)^{\prime}=(1-\beta / \alpha) w^{-\beta / \alpha}(t) w^{\prime}(t) \leq-\frac{(1-\beta / \alpha)}{\left(2 \delta^{1 / \alpha}\right)^{\beta}} \sum_{i=1}^{m} q_{i}(t) H\left(\delta^{1 / \alpha} R\left(t-\sigma_{i}\right)\right)$. 
Integrating this inequality form $t_{5}$ to $t$, we have

$$
\left[w^{1-\beta / \alpha}(\eta)\right]_{t_{5}}^{t} \leq-\frac{(1-\beta / \alpha)}{\left(2 \delta^{1 / \alpha}\right)^{\beta}} \int_{t_{5}}^{t} \sum_{i=1}^{m} q_{i}(\eta) H\left(\delta^{1 / \alpha} R\left(\eta-\sigma_{i}\right)\right) \mathrm{d} \eta .
$$

Since $\beta / \alpha<1$ and $w(t)$ is positive and non-increasing, we have

$$
\int_{t_{2}}^{t} \sum_{i=1}^{m} q_{i}(\eta) H\left(\delta^{1 / \alpha} R\left(\eta-\sigma_{i}\right)\right) \mathrm{d} \eta \leq \frac{\left(2 \delta^{1 / \alpha}\right)^{\beta}}{(1-\beta / \alpha)} w^{1-\beta}\left(t_{5}\right)<\infty .
$$

This contradicts (3.1).

If $x(t)<0$ for $t \geq t_{1}$, then we set $y(t):=-x(t)$ for $t \geq t_{1}$ in (1.1). Using (A1), we find

$$
\frac{\mathrm{d}}{\mathrm{d} t}\left[r(t)\left[\frac{\mathrm{d}}{\mathrm{d} t}(y(t)+p(t) y(t-\tau))\right]^{\alpha}\right]+\sum_{i=1}^{m} q_{i}(t) G\left(y\left(t-\sigma_{i}\right)\right)=0 \text { for } t \geq t_{1},
$$

where $G(u)=-H(-u)$ and $G$ is also satisfies (A1). Then, proceeding as above, we find the same contradiction. This proves the oscillation of all solutions

Next, we show that (3.1) is necessary. Suppose that (3.1) does not hold; so for some $\delta>0$ the integral in (3.1) is finite. Then there exists $T \geq \sigma$ such that

$$
\int_{T}^{\infty} \sum_{i=1}^{m} q_{i}(\eta) H\left(\delta^{1 / \alpha} R\left(\eta-\sigma_{i}\right)\right) \mathrm{d} \eta \leq \delta / 3
$$

Let us consider the closed subset of continuous functions

$$
\begin{aligned}
M=\{x \in C([T-\sigma,+\infty), \mathbb{R}): & \\
& \left.(\delta / 3)^{1 / \alpha}[R(t)-R(T)] \leq x(t) \leq \delta^{1 / \alpha}[R(t)-R(T)]\right\} .
\end{aligned}
$$

Then we define the operator $\Phi: M \rightarrow C([T-\sigma,+\infty), \mathbb{R})$ by

$$
(\Phi x)(t)= \begin{cases}(\Phi x)(T), & t \in[T-\sigma, T), \\ -p(t) x(t-\tau)+ & \\ \int_{T}^{t}\left[\frac{1}{r(\eta)}\left[\delta / 3+\int_{\eta}^{\infty} \sum_{i=1}^{m} q_{i}(\zeta) H\left(x\left(\zeta-\sigma_{i}\right)\right) \mathrm{d} \zeta\right]\right]^{1 / \alpha} \mathrm{d} \eta, & t \geq T .\end{cases}
$$

For $x \in M$ and $t \geq T$, we have

$$
\begin{aligned}
(\Phi x)(t) & \geq \int_{T}^{t}\left[\frac{1}{r(\eta)}\left[\delta / 3+\int_{\eta}^{\infty} \sum_{i=1}^{m} q_{i}(\zeta) H\left(x\left(\zeta-\sigma_{i}\right)\right) \mathrm{d} \zeta\right]\right]^{1 / \alpha} \mathrm{d} \eta \\
& \geq \int_{T}^{t}\left[\frac{1}{r(\eta)} \frac{\delta}{3}\right]^{1 / \alpha} \mathrm{d} \eta=(\delta / 3)^{1 / \alpha}[R(t)-R(T)] .
\end{aligned}
$$


For $x \in M$ and $t \geq T$, we have $x(t) \leq \delta^{1 / \alpha} R(t)$ and $H(x) \leq H\left(\delta^{1 / \alpha}(R(t))\right)$. Then using (3.8) and (A3) we have

$$
\begin{aligned}
(\Phi x)(t) & \leq-p(t) x(t-\tau)+\int_{T}^{t}\left[\frac{1}{r(\eta)}(\delta / 3+\delta / 3)\right]^{1 / \alpha} \mathrm{d} \eta \\
& \leq a \delta^{1 / \alpha}[R(t-\tau)-R(T)]+(2 \delta / 3)^{1 / \alpha}[R(t)-R(T)] \\
& \leq a \delta^{1 / \alpha}[R(t)-R(T)]+(2 \delta / 3)^{1 / \alpha}[R(t)-R(T)] \\
& =\left(a+(2 / 3)^{1 / \alpha}\right) \delta^{1 / \alpha}[R(t)-R(T)] \\
& \leq \delta^{1 / \alpha}[R(t)-R(T)] .
\end{aligned}
$$

Thus $\Phi x \in M$. Define $u_{n}:[T-\sigma,+\infty) \rightarrow \mathbb{R}$ by the recursive formula

$$
\begin{gathered}
u_{1}(t)=\left\{\begin{array}{lr}
0, & t \in[t-\sigma, T], \\
(\delta / 3)^{1 / \alpha}[R(t)-R(T)], & t \geq T .
\end{array}\right. \\
u_{n}(t)=\left(\Phi u_{n-1}\right)(t) \text { for } n>1 .
\end{gathered}
$$

Using that $H$ is non-decreasing it is easy to verify that for $n>1$

$$
(\delta / 3)^{1 / \alpha}[R(t)-R(T)] \leq u_{n-1}(t) \leq u_{n}(t) \leq \delta^{1 / \alpha}[R(t)-R(T)] .
$$

Therefore, the pointwise limit of the sequence exists. Let $\lim _{n \rightarrow \infty} u_{n}(t)=u(t)$ for $t \geq T-\sigma$. By Lebesgue's dominated convergence theorem $u \in M$ and $(\Phi u)(t)=u(t)$, where $u(t)$ is a solution of equation (1.1) on $[T-\sigma, \infty)$. Hence, (3.1) is a necessary condition. This completes the proof.

TheOREM 3.2. Under assumptions (A1)-(A3) and (2.3), every solution of (1.1) oscillates or converges to zero if and only if (3.1) holds for every $\delta>0$.

Pr o of. To prove sufficiency by contradiction, we assume that $x$ is an eventually positive solution of (1.1) which does not converges to zero. Then, there exists $t_{1} \geq t_{0}$ such that $x(t)>0, x(t-\tau)>0$ and $x\left(t-\sigma_{i}\right)>0$ for $t \geq t_{1}$ and $i=1,2, \ldots, m$. Then we have (2.1). From Lemma 2.1, $z$ satisfies one of the cases $(\mathrm{C} 1)$ and $(\mathrm{C} 2)$ for $t \geq t_{2}$, where $t_{2} \geq t_{1}$. We consider each of two cases separately.

Case 1. Let $z$ satisfies $(C 1)$ for $t \geq t_{2}$. Therefore,

$$
\begin{aligned}
0 & \geq \lim _{t \rightarrow \infty} z(t)=\limsup _{t \rightarrow \infty} z(t) \geq \limsup _{t \rightarrow \infty}(x(t)-a x(t-\tau)) \\
& \geq \limsup _{t \rightarrow \infty} x(t)+\liminf _{t \rightarrow \infty}(-a x(t-\tau))=(1-a) \limsup _{t \rightarrow \infty} x(t)
\end{aligned}
$$

implies that $\limsup _{t \rightarrow \infty} x(t)=0$ and hence $\lim _{t \rightarrow \infty} x(t)=0$, which contradicts the assumption that $x$ does not converges to zero.

Case 2. Let $z$ satisfies $(\mathrm{C} 2)$ for $t \geq t_{2}$. The case follows from Theorem 3.1 . Hence, (3.1) is a sufficient condition. 


\section{NECESSARY AND SUFFICIENT CONDITIONS ...}

The case where $x$ is negative solution is similar and we omit it here.

The necessary part is the same as in the Theorem 3.1. Thus, the proof of the theorem is complete.

TheOREM 3.3. Under assumptions (A1), (A2), (A4), (2.4) and $r(t)>0$ on $\left[-\sigma_{j}, \infty\right)$, every solution of (1.1) either oscillates or converges to zero if

$$
\int_{0}^{\infty}\left[\frac{1}{r\left(\eta-\sigma_{j}\right)} \int_{\eta}^{\infty} q_{j}(\zeta) \mathrm{d} \zeta\right]^{1 / \alpha} \mathrm{d} \eta=+\infty \quad \text { for some } j .
$$

Pr o of. To prove it by contradiction, suppose that $x$ is an eventually positive solution of (1.1) which does not converges to zero and we use same type of argument as in the proof of Theorem 3.2 for the case (C1). Let us consider $z$ satisfies (C2) for $t \geq t_{2}$. By Remark 2.1, there exists a constant $\delta>0$ and $t_{3} \geq t_{2}$ such that $z\left(t-\sigma_{i}\right) \geq \delta$ for $t \geq t_{3}$ and $i=1,2, \ldots, m$.

Upon using $z(t) \leq x(t)$ and by assumption (2.4), we have

$$
H\left(x\left(t-\sigma_{i}\right)\right) \geq H\left(z\left(t-\sigma_{i}\right)\right)=\frac{H\left(z\left(t-\sigma_{i}\right)\right)}{z^{\beta}\left(t-\sigma_{i}\right)} z^{\beta}\left(t-\sigma_{i}\right) \geq \frac{H(\delta)}{\delta^{\beta}} z^{\beta}\left(t-\sigma_{i}\right) .
$$

Integrating (1.1) from $t$ to $\infty$, we have

$$
\lim _{A \rightarrow \infty}\left[r(\eta)\left(z^{\prime}(\eta)\right)^{\alpha}\right]_{t}^{A}+\int_{t}^{\infty} \sum_{i=1}^{m} q_{i}(\eta) \frac{H(\delta)}{\delta^{\beta}} z^{\beta}\left(\eta-\sigma_{i}\right) \mathrm{d} \eta \leq 0 .
$$

Using that $r(t)\left(z^{\prime}(t)\right)^{\alpha}$ is positive and non-increasing, we have

$$
\int_{t}^{\infty} \sum_{i=1}^{m} q_{i}(\eta) \frac{H(\delta)}{\delta^{\beta}} z^{\beta}\left(\eta-\sigma_{i}\right) \mathrm{d} \eta \leq r(t)\left(z^{\prime}(t)\right)^{\alpha} \leq r\left(t-\sigma_{j}\right)\left(z^{\prime}\left(t-\sigma_{j}\right)\right)^{\alpha}
$$

for all $t \geq t_{3}$ and all $j$ in $\{1, \ldots, m\}$. Therefore,

$$
\left[\frac{1}{r\left(t-\sigma_{j}\right)} \int_{t}^{\infty} \sum_{i=1}^{m} q_{i}(\eta) \frac{H(\delta)}{\delta^{\beta}} z^{\beta}\left(\eta-\sigma_{i}\right) \mathrm{d} \eta\right]^{1 / \alpha} \leq z^{\prime}\left(t-\sigma_{j}\right) .
$$

Dividing by $z^{\beta / \alpha}\left(t-\sigma_{j}\right)$ and then integrating from $t_{3}$ to $\infty$, we have

$$
\left(\frac{H(\delta)}{\delta^{\beta}}\right)^{1 / \alpha} \int_{t_{3}}^{\infty}\left[\frac{1}{r\left(\eta-\sigma_{j}\right)} \int_{\eta}^{\infty} \sum_{i=1}^{m} q_{i}(\zeta) \frac{z^{\beta}\left(\zeta-\sigma_{i}\right)}{z^{\beta}\left(\eta-\sigma_{j}\right)} \mathrm{d} \zeta\right]^{1 / \alpha} \mathrm{d} \eta \leq \int_{t_{3}}^{\infty} \frac{z^{\prime}\left(\eta-\sigma_{j}\right)}{z^{\beta / \alpha}\left(\eta-\sigma_{j}\right)} \mathrm{d} \eta .
$$

Since $z$ is increasing, for $\zeta \geq \eta$ we have $z^{\beta}\left(\zeta-\sigma_{i}\right) \geq z^{\beta}\left(\eta-\sigma_{i}\right)$. Note that the summands $z^{\beta}\left(\eta-\sigma_{i}\right) / z^{\beta}\left(\zeta-\sigma_{j}\right)$ are positive for all $i, j$, and equal 1 when $i=j$. 
Then considering only the summand when $i=j$, integrating on the right-hand side, and using that the integrand is positive, we have

$$
\left(\frac{H(\delta)}{\delta^{\beta}}\right)^{1 / \alpha} \int_{t_{3}}^{\infty}\left[\frac{1}{r\left(\eta-\sigma_{j}\right)} \int_{\eta}^{\infty} q_{i}(\zeta) \mathrm{d} \zeta\right]^{1 / \alpha} \mathrm{d} \eta \leq \frac{z^{1-\beta / \alpha}\left(t_{3}-\sigma_{j}\right)}{\beta / \alpha-1}<\infty .
$$

This contradicts (3.9). The case where $x$ is eventually negative solution is omitted since it can be dealt similarly. This proves the oscillation of all solutions.

Theorem 3.4. Assume that (A1), (A2) and (A4) hold. If

$$
\int_{0}^{\infty}\left[\frac{1}{r(\eta)} \int_{\eta}^{\infty} \sum_{i=1}^{m} q_{i}(\zeta) \mathrm{d} \zeta\right]^{1 / \alpha} \mathrm{d} \eta<\infty
$$

holds, then (1.1) admits a positive bounded solution.

Proof. Due to (3.12), it is possible to find $T \geq \sigma$ such that

$$
\int_{T}^{\infty}\left[\frac{1}{r(\eta)} \int_{\eta}^{\infty} \sum_{i=1}^{m} q_{i}(\zeta) \mathrm{d} \zeta\right]^{1 / \alpha} \mathrm{d} \eta \leq \frac{1-a}{5(H(1))^{1 / \alpha}}, \quad \delta>0 .
$$

Let us consider the closed subset of continuous functions

$$
M=\left\{x \in C([T-\sigma,+\infty), \mathbb{R}): \frac{1-a}{5} \leq x(t) \leq 1\right\} .
$$

Then we define the operator $\Phi: M \rightarrow C([T-\sigma,+\infty), \mathbb{R})$ by

$$
(\Phi x)(t)=\left\{\begin{array}{lr}
(\Phi x)(T), & t \in[t-\sigma, T) \\
-p(t) x(t-\tau)+\frac{1-a}{5} & t \geq T . \\
+\int_{T}^{t}\left[\frac{1}{r(\eta)} \int_{\eta}^{\infty} \sum_{i=1}^{m} q_{i}(\zeta) H\left(x\left(\zeta-\sigma_{i}\right)\right) \mathrm{d} \zeta\right]^{1 / \alpha} \mathrm{d} \eta, & t \geq .
\end{array}\right.
$$

Note that for $x \in M$ and $t \geq T$, we have $(\Phi x)(t) \geq \frac{1-a}{5}$. Also for $x \in M$ and $t \geq T$, we have

$$
\begin{aligned}
(\Phi x)(t) & \leq a+\frac{1-a}{5}+(H(1))^{1 / \alpha} \int_{T}^{t}\left[\frac{1}{r(\eta)} \int_{\eta}^{\infty} \sum_{i=1}^{m} q_{i}(\zeta) \mathrm{d} \zeta\right]^{1 / \alpha} \mathrm{d} \eta \\
& \leq a+\frac{1-a}{5}+\frac{1-a}{5}=\left(\frac{3 a+2}{5}\right)<1 .
\end{aligned}
$$

Thus $\Phi x \in M$. The rest of the proof follows from Theorem 3.1. This completes the proof of the theorem. 


\section{NECESSARY AND SUFFICIENT CONDITIONS ...}

We illustrate our main results with the next two examples.

ExAmPle 3.1. Consider the delay differential equation

$$
\begin{array}{r}
\left(e^{-t}(x(t)+p(t) x(t-\tau))^{5 / 7}\right)^{\prime}+\frac{1}{t+1}(x(t-2))^{1 / 3}+\frac{1}{t+2}(x(t-1))^{1 / 3}=0 \\
t \geq 0
\end{array}
$$

Here
$\alpha=5 / 7, r(t)=e^{-t}, p(t)=-e^{-t}, R(t)=\int_{0}^{t} e^{7 s / 5} \mathrm{~d} s=\frac{5}{7}\left(e^{7 t / 5}-1\right)$ and $i=1,2$.

$H(u)=u^{1 / 3}$. For $\beta=1 / 2$, we have $H(u) / u^{\beta}=u^{-1 / 6}$ which is a decreasing function. To check (3.1) we have

$$
\begin{aligned}
\int_{0}^{\infty} \sum_{i=1}^{m} q_{i}(\eta) H\left(\delta^{1 / \alpha} R\left(\eta-\sigma_{i}\right)\right) \mathrm{d} \eta & \geq \int_{0}^{\infty} q_{1}(\eta) H\left(\delta^{1 / \alpha} R\left(\eta-\sigma_{1}\right)\right) \mathrm{d} \eta \\
& \geq \int_{0}^{\infty} \frac{1}{\eta+1}\left(\delta^{7 / 5} \frac{5}{7}\left(e^{7(\eta-2) / 5}-1\right)\right)^{1 / 3} \\
\mathrm{~d} \eta & =\infty \quad \forall \delta>0,
\end{aligned}
$$

because the integrand approaches $+\infty$ as $\eta \rightarrow+\infty$. So that all the assumptions in Theorem 3.1 hold; hence every unbounded solution of (3.14) oscillates.

EXAMPLE 3.2. Consider the delay differential equation

$$
\begin{array}{r}
\left(e^{-t}(x(t)+p(t) x(t-\tau))^{3 / 5}\right)^{\prime}+\frac{1}{(t+1)^{2}}(x(t-2))^{5 / 3}+\frac{1}{(t+2)^{2}}(x(t-1))^{5 / 3}=0 \\
t \geq 0 .
\end{array}
$$

Here
$\alpha=3 / 5, r(t)=e^{-t}, p(t)=-e^{-t}, R(t)=\int_{0}^{t} e^{5 s / 3} \mathrm{~d} s=\frac{3}{5}\left(e^{5 t / 3}-1\right)$ and $i=1,2$.

$H(u)=u^{5 / 3}$. For $\beta=4 / 3$, we have $H(u) / u^{\beta}=u^{1 / 3}$ which is an increasing function. The integral in (3.9) is greater than or equal to

$$
\int_{2}^{\infty}\left[e^{\eta-\sigma_{1}} \int_{\eta}^{\infty} \frac{1}{(\zeta+1)^{2}}\right]^{5 / 3} \mathrm{~d} \eta=\int_{2}^{\infty}\left[e^{\eta-2} \frac{1}{\eta+1}\right]^{5 / 3} \mathrm{~d} \eta=\infty
$$

because the integrand approaches $+\infty$ as $\eta \rightarrow+\infty$. So that all the assumptions in Theorem 3.3 hold; hence every solution of (3.15) either oscillates or converges to zero. 


\section{SHYAM SUNDAR SANTRA}

\section{Final comment}

It is worth observation that we have established the oscillation of all solutions of the nonlinear equation (1.1), when $-1<p(t) \leq 0$. We failed to obtain the necessary and sufficient conditions in the other ranges of $p$. Therefore, the undertaken problem is incomplete for all range of $p(t)$.

Here, we will be giving two remarks and two examples to conclude the paper.

Remark 4.1. The Banach's contraction principle can be applied for the Theorem 3.3 .

Remark 4.2. The results of this paper also hold for equations of the form

$$
\frac{\mathrm{d}}{\mathrm{d} t}\left[r(t)\left[\frac{\mathrm{d}}{\mathrm{d} t}(x(t)+p(t) x(t-\tau))\right]^{\alpha}\right]+\sum_{i=1}^{m} q_{i}(t) H_{i}\left(x\left(t-\sigma_{i}\right)\right)=0 .
$$

In order to extend Theorem 3.1-Theorem 3.3, there exists an index $i$ such that $H_{i}(i=1,2, \ldots, m)$ fulfills (A1)-(A4), (2.3), (2.4) and (3.1).

We finalize the paper by presenting two examples, which show how Remark 4.2 can be applied.

ExAmple 4.1. Consider the delay differential equation

$$
\begin{array}{r}
\left(e^{-t}(x(t)+p(t) x(t-\tau))^{3 / 5}\right)^{\prime}+\frac{1}{t+1}(x(t-2))^{1 / 3}+\frac{1}{t+2}(x(t-1))^{1 / 5}=0 \\
t \geq 0
\end{array}
$$

$\alpha=3 / 5, r(t)=e^{-t}, p(t)=-e^{-t}, R(t)=\int_{0}^{t} e^{5 s / 3} \mathrm{~d} s=\frac{3}{5}\left(e^{5 t / 3}-1\right)$ and $i=1,2$.

$H_{1}(u)=u^{1 / 3}$ and $H_{2}(u)=u^{1 / 5}$. For $\beta=1 / 2$, we have $H_{1}(u) / u^{\beta}=u^{-1 / 6}$ and $H_{2}(u) / u^{\beta}=u^{-3 / 10}$ which both are decreasing functions. To check (3.4) we have

$$
\begin{aligned}
& \int_{0}^{\infty} \sum_{i=1}^{m} q_{i}(\eta) H_{i}\left(\delta^{1 / \alpha} R\left(\eta-\sigma_{i}\right)\right) \mathrm{d} \eta \geq \int_{0}^{\infty} q_{1}(\eta) H_{1}\left(\delta^{1 / \alpha} R\left(\eta-\sigma_{1}\right)\right) \mathrm{d} \eta \\
& =\int_{0}^{\infty} \frac{1}{\eta+1}\left(\delta^{5 / 3} \frac{3}{5}\left(e^{5(\eta-2) / 3}-1\right)\right)^{1 / 3} \mathrm{~d} \eta=\infty \quad \forall \delta>0,
\end{aligned}
$$

because the integrand approaches $+\infty$ as $\eta \rightarrow+\infty$. So that all the assumptions in Theorem 3.1 hold; hence every unbounded solution of (4.1) oscillates. 


\section{NECESSARY AND SUFFICIENT CONDITIONS ...}

EXAMPLE 4.2. Consider the delay differential equation

$$
\begin{array}{r}
\left(e^{-t}(x(t)+p(t) x(t-\tau))^{5 / 7}\right)^{\prime}+\frac{1}{(t+1)^{2}}(x(t-2))^{5 / 3}+\frac{1}{(t+2)^{2}}(x(t-1))^{3}=0 \\
t \geq 0
\end{array}
$$

Here

$$
\alpha=5 / 7, r(t)=e^{-t}, r(t)=-e^{-t}, R(t)=\frac{5}{7}\left(e^{7 t / 5}-1\right) .
$$

$H_{1}(u)=u^{5 / 3}$ and $H_{2}(u)=u^{3}$. For $\beta=4 / 3$, we have $H_{1}(u) / u^{\beta}=u^{1 / 3}$ and $H_{2}(u) / u^{\beta}=u^{5 / 3}$ which both are increasing functions. Clearly, all the assumptions in Theorem 3.3 hold; hence every solution of (4.2) either oscillates or converges to zero.

Acknowledgement. The author is thankful to the referee for his valuable comments and suggestions in the completion of this paper.

\section{REFERENCES}

[1] AGARWAL, R.P.-BOHNER, M.-LI, T.- ZHANG, C.: Oscillation of second order differential equations with a sublinear neutral term, Carpathian J. Math. 30 (2014), 1-6.

[2] BRANDS, J. J. M. S.: Oscillation theorems for second-order functional-differential equations, J. Math. Anal. Appl. 63 (1978), no. 1, 54--64.

[3] BACULIKOVA, B.-DZURINA, J.: Oscillation theorems for second order neutral differential equations, Comput. Math. Appl. 61 (2011), 94-99.

[4] Oscillation theorems for second order nonlinear neutral differential equations, Comput. Math. Appl. 62 (2011), 4472-4478.

[5] BACULIKOVA, B.- LI, T.-DZURINA, J.: Oscillation theorems for second order neutral differential equations, Electron. J. Quali. Theo. Diff. Eqn. 74 (2011), 1-13.

[6] DŽURINA, J: Oscillation theorems for second order advanced neutral differential equations, Tatra Mt. Math. Publ., 48 (2011), 61-71.

[7] HALE, J.: Theory of Functional Differential Equations. In: Applied Mathematical Sciences, 2nd ed. Vol. 3, Springer-Verlag, Berlin, 1977.

[8] KARPUZ, B.-SANTRA, S.S.: Oscillation theorems for second-order nonlinear delay differential equations of neutral type, Hacettepe J. Math. Stat., 48 (2019), no. 3. 633-643.

[9] LI, T.-ROGOVCHENKO, Y. V.: Oscillation theorems for second order nonlinear neutral delay differential eqquations, Abstr. Appl. Anal. 2014 (2014), ID 594190, 1-5.

[10] PINELAS, S.-SANTRA, S. S.: Necessary and sufficient condition for oscillation of nonlinear neutral first-order differential equations with several delays, J. Fixed Point Theory Appl. 20 (2018) no. 27; https://doi.org/10.1007/s11784-018-0506-9 


\section{SHYAM SUNDAR SANTRA}

[11] QIAN, Y.-XU, R.: Some new osciilation criteria for higher order quasi-linear neutral delay differential equations, Differ. Equ. Appl. 3 (2011), no. 3, 323-335.

[12] SANTRA, S. S.: Existence of positive solution and new oscillation criteria for nonlinear first order neutral delay differential equations, Differ. Equ. Appl. 8 (2016), no. 1, 33-51.

[13] SANTRA, S. S.: Oscillation analysis for nonlinear neutral differential equations of second order with several delays, Mathematica, 59(82)(1-2): (2017), 111-123.

[14] SANTRA, S. S.: Oscillation analysis for nonlinear neutral differential equations of second order with several delays and forcing term, Mathematica, 61(84) (2019), no. 1, 63-78.

[15] TRIPATHY, A. K.-PANDA, B.-SETHI, A. K.: On oscillatory nonlinear second order neutral delay differential equations, Differ. Equ. Appl. 8 (2016), no. 2, 247-258.

[16] WONG, J. S. W.: Necessary and suffcient conditions for oscillation of second order neutral differential equations, J. Math. Anal. Appl. 252 (2000), no. 1, 342-352.

[17] YANG,Q-XU, Z.: Oscillation criteria for second order quasi-linear neutral delay differential equations on time scales, Comput. Math. Appl. 62 (2011), 3682-3691.

[18] YE, L.-XU, Z.: Oscillation criteria for second order quasilinear neutral delay differential equations, Appl. Math. Comput. 207 (2009), 388-396.

Received November 11, 2018

Department of Mathematics
JIS College of Engineering
Kalyani-741235
Nadia, West Bengal
INDIA
E-mail: shyam01.math@gmail.com 